\title{
Expression of Matrix Metalloproteinases and Tissue Inhibitors of Metalloproteinases in Gliomatosis Peritonei Derived from Ovarian Immature Teratoma
}

\author{
Yasuhiko Kiyozuka1, Yasunori Muraoka², Kazuo Imamura ${ }^{2}$, Hideto Senzaki ${ }^{1}$, \\ Yoshiko Uemura $^{1}$, Airo Tsubura ${ }^{1}$ and Michiaki Yakushijii ${ }^{2}$ \\ ${ }^{1}$ Department of Pathology II, Kansai Medical University, 10-15 Fumizono, Moriguchi, Osaka 570-8506 and ${ }^{2}$ Depart- \\ ment of Obstetrics and Gynecology, Kurume University School of Medicine, 67 Asahi, Kurume, Fukuoka 830-0011
}

Received for publication September 16, 1997

\begin{abstract}
The mechanisms of gliomatosis peritonei, composed exclusively of mature glial tissue implants on the peritoneum or omentum, are not yet understood. In the present study, the expressions of metalloproteinases (MMPs; MMP-1, -2, -3, and -9) and tissue inhibitors of metalloproteinases (TIMPs; TIMP1 and -2) were examined in ovarian immature teratomas with and without glial implants to evaluate the roles of MMPs and TIMPs in gliomatosis peritonei. The immunostaining showed a high expression of MMP-1 in the glial fibrillary acidic protein (GFAP)-positive cells in the glial implants, and in the primary immature teratomas regardless of the ex-
\end{abstract}

istence of gliomatosis peritonei. A weak expression of MMP-2 was seen in both primary and implant sites in the case with gliomatosis peritonei, but not in the cases without glial implants. In contrast, a high TIMP-2 expression was seen in the glial cells in the cases without gliomatosis peritonei but was absent in the primary as well as peritoneal implants in the case with gliomatosis peritonei. The expressions of MMP-3, MMP-9, and TIMP-1 were universally negative. These results suggest that the loss of TIMP-2 plays a crucial role in the development of glial implants of ovarian immature teratoma.

Key words: Gliomatosis peritonei, Ovarian teratoma, Matrix metalloproteinase, Tissue inhibitor of metalloproteinase

\section{Introduction}

Gliomatosis peritonei, i.e., peritoneal implants of mature glial cells, is a rare event. Only thirty-six cases appear in the literature compiled on the Medline [1, 3-6, 8, 9, 13, 19]. The mode of dissemination of gliomatosis peritonei is hypothesized to be an intraperitoneal spread via a capsular defect of the primary tumor, either spontaneously or surgically $[14,17,19]$. However, it is not known why only glial tissues in some patients show peritoneal implants, or how the glial cells detach from the primary site, migrate, and implant in the abdominal cavity. The mechanism of this glial cell implantation is largely unknown. Regarding the mechanism of tumor cell detach-

Selected by "The 10th International Congress of Histochemistry and Cytochemistry" held in Kyoto, Japan on Aug. 18-23, 1996.

Correspondence to: Yasuhiko Kiyozuka M.D., Ph.D., Department of Pathology II, Kansai Medical University, 10-15 Fumizono-cho, Moriguchi-shi, Osaka 570-8506, Japan. ment from the primary site, Liotta et al. [11] proposed the following three-step theory; (i) tumor cells attach to the extracellular matrix (ECM), (ii) then secrete enzymes that locally degrade the ECM, (iii) followed by the migration of tumor cells into the ECM.

Matrix metalloproteinases (MMPs) play an important role in these steps by degrading and remodeling the ECM $[12,16,20]$. The regulation of MMPs by tissue inhibitors of metalloproteinases (TIMPs) combined with other inhibitors is essential to tumor invasion [20]. At least 14 members of the MMP family and 3 different TIMP molecules have been described. Based on their substrate preferences, MMP-1 is classified as a collagenase, MMP-2 as a collagenase/gelatinase A, MMP-3 as a stromelysin, and MMP-9 as a gelatinase B [20]. The activities of MMPs are closely regulated by TIMPs. In general, the substrate preference of TIMP-1 shows a higher affinity to interstitial collagenase such as MMP-1, and TIMP-2 shows a higher affinity toward gelatinases such as MMP-2 and -9 [20]. 
Several studies have reported a correlation between MMP production and the concomitant expression of TIMPs in several glioma cell lines $[2,7,10,18]$. The participation of MMPs and TIMPs in the mechanism of gliomatosis peritonei is thus of interest. The present study was conducted to examine the expressions of MMPs and TIMPs in a case of gliomatosis peritonei derived from an immature ovarian teratoma, in comparison to those in cases without glial implants.

\section{Materials and Methods}

\section{A case of gliomatosis peritonei}

A 24-year-old, primipara woman was incidentally noted to have tumors in both ovaries during a Cesarean section due to cephalo-pelvic disproportion. The right ovarian tumor was of new-born-child-head (NBCH) size and the left ovarian tumor was $5 \mathrm{~cm}$ in diameter. A rightoophorectomy and a left-enucleation was performed. According to Norris et al. [15], the histological evaluation of the right ovarian tumor revealed a grade 2 immature teratoma. The left ovarian tumor was pathologically diagnosed as a mature cystic teratoma. The patient's serum AFP (19.2 ng/ml), CA125 (98 U/ml) and CA19-9 $(39.4 \mathrm{U} / \mathrm{ml})$ levels were elevated. In the staging laparotomy $\left(\mathrm{pT}_{3} \mathrm{~N}_{1} \mathrm{M}_{0}\right)$, followed by Cesarean section, multiple firm gray-white $0.2-1.0 \mathrm{~cm}$ nodules composed exclusively of mature (grade 0 ) glial nodules were present on the diaphragm, omentum, the posterior wall of the peritoneum and Douglas' cul-de-sac. An omentectomy and pelvic-to-paraaortic lymphadnectomy were performed. Postoperatively, the patient received three rounds of chemotherapy with the PEP combination (cisplatinum, etoposide, and pepleomycin) and has been well for six months after the operation.

\section{Immunohistochemical study}

The formalin-fixed and paraffin-embedded surgical specimens of ovarian sections including glial tissue, and sections of the gliomatosis peritonei were examined. Two cases of immature teratoma with comparable grades of differentiation, both containing glial tissues (from 6- and 13-year-old females, respectively) without gliomatosis peritonei were used for comparison. Sections cut at $4 \mu \mathrm{m}$
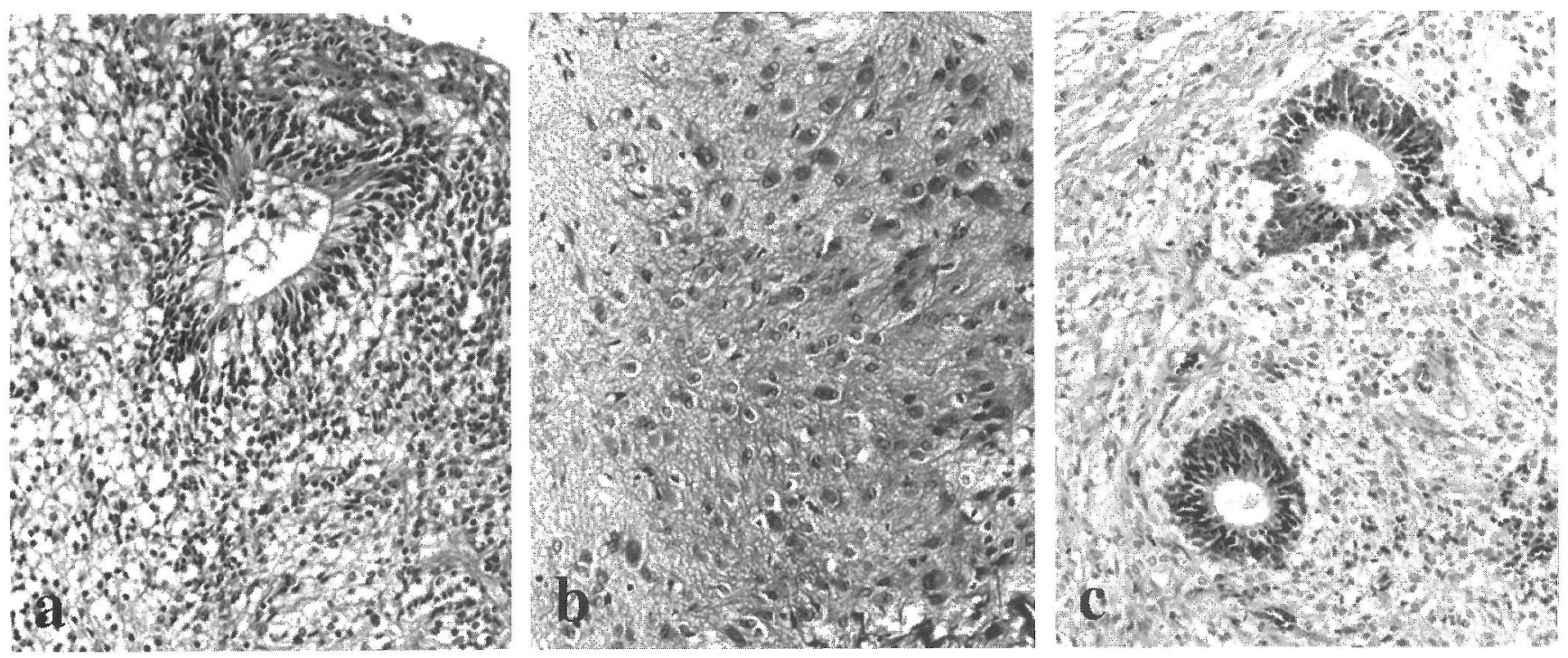

Fig. 1. (a) Primary tumor of the immature teratoma of the ovary with gliomatosis peritonei. It is composed predominantly of neuroectodermal tissues and immature glial cells $(\mathrm{H} \& \mathrm{E}, \times 200)$. (b) Peritoneal implant. The glial tissue is composed mainly of astrocytes and oligodendroglias comprising a fibrillary network $(\mathrm{H} \& \mathrm{E}, \times 200)$. (c) Immature teratoma without gliomatosis peritonei. Immature glial component is admixed with neuroectodermal tissues $(\mathrm{H} \& \mathbb{E}, \times 200)$.

Table 1. MMPs and TIMPs expression in immature teratomas with and without gliomatosis peritonei

\begin{tabular}{lcccccc}
\hline Immature teratoma & MMP-1 & MMP-2 & MMP-3 & MMP-9 & TIMP-1 & TIMP-2 \\
\hline $\begin{array}{l}\text { with gliomatosis peritonei* } \\
(\mathrm{n}=1)\end{array}$ & + & $\pm /-$ & - & - & - & - \\
$\begin{array}{l}\text { without gliomatosis peritonei } \\
(\mathrm{n}=2)\end{array}$ & + & - & - & - & - & + \\
\end{tabular}

* Expression is identical in primary and glial implants. + strong, \pm weak, - negative 
were deparaffinized and treated with 3\% hydrogen peroxide in methanol $(10 \mathrm{~min})$ to block endogenous peroxidase. The primary antibodies were applied for $1 \mathrm{hr}$ at room temperature. After the sections were washed in phosphate-buffered saline (PBS), immunostaining by the labeled streptavidin biotin (LSAB) method using a Dako LSAB Kit (Dako, Carpinteria, CA, USA) was performed, and the reaction products were visualized with freshly prepared $0.01 \%$ 3, 3'-diaminobenzidine tetrahydrochloride (DAB, Wako Pure Chemicals, Osaka, Japan). The MMP-1, -2, -3, and -9, and TIMP-1, and -2 expressions were identified using the respective mouse monoclonal anti-human MMP or TIMP antibodies (each purchased from Fuji Chemical Ind., Toyama, Japan). Monoclonal anti-glial fibrillary acidic protein (GFAP) antibody (Dako) was used to assist the identification of glial tissues. Replacement of the primary antibodies with normal mouse serum served as the negative control.

\section{Results}

Pathology of primary ovarian tumor and glial implants

The ovarian tumor (grade 2) showing gliomatosis peritonei contained foci of primitive neuroectodermal
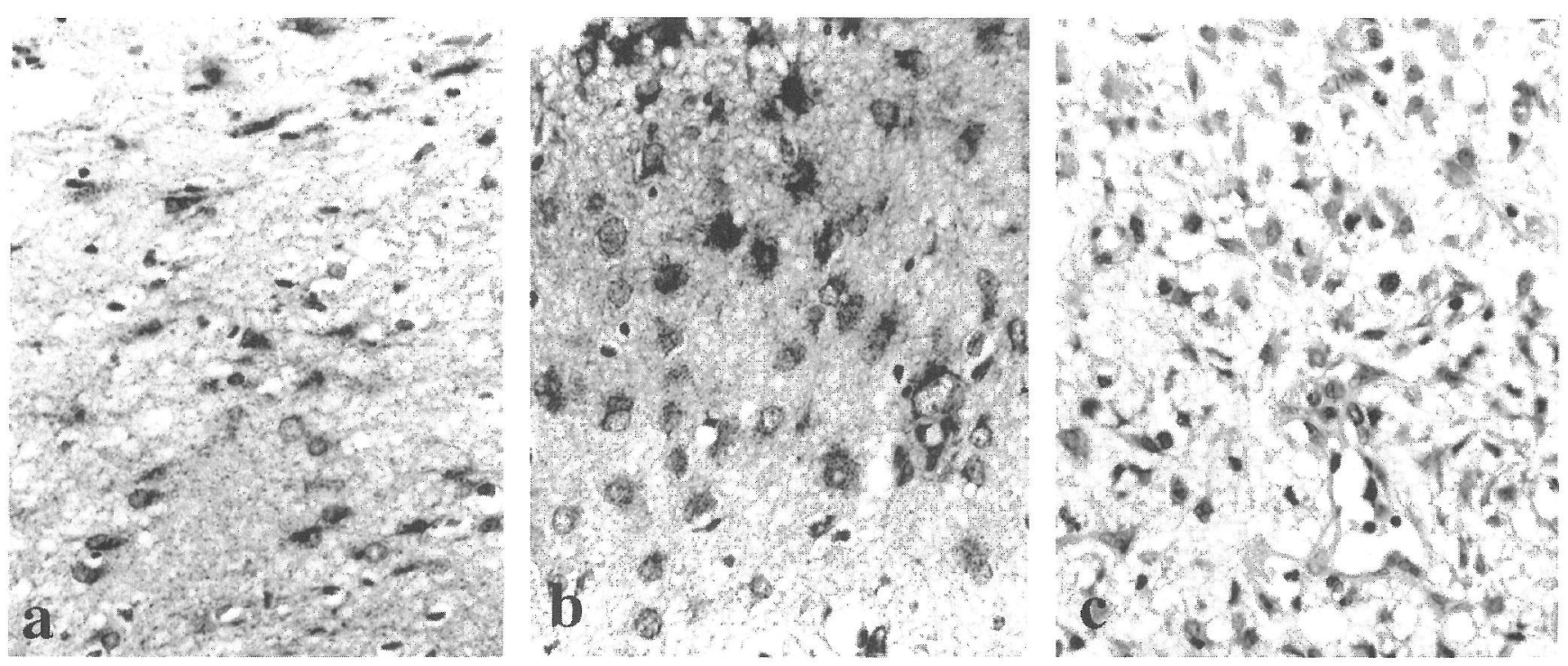

Fig. 2. Positive MMP-1 expression. (a) glial cells in immature ovarian teratoma with glial implants, (b) glial implants, and (c) immature teratoma without gliomatosis peritonei $(\mathrm{MMP}-1$, each $\times 200)$.

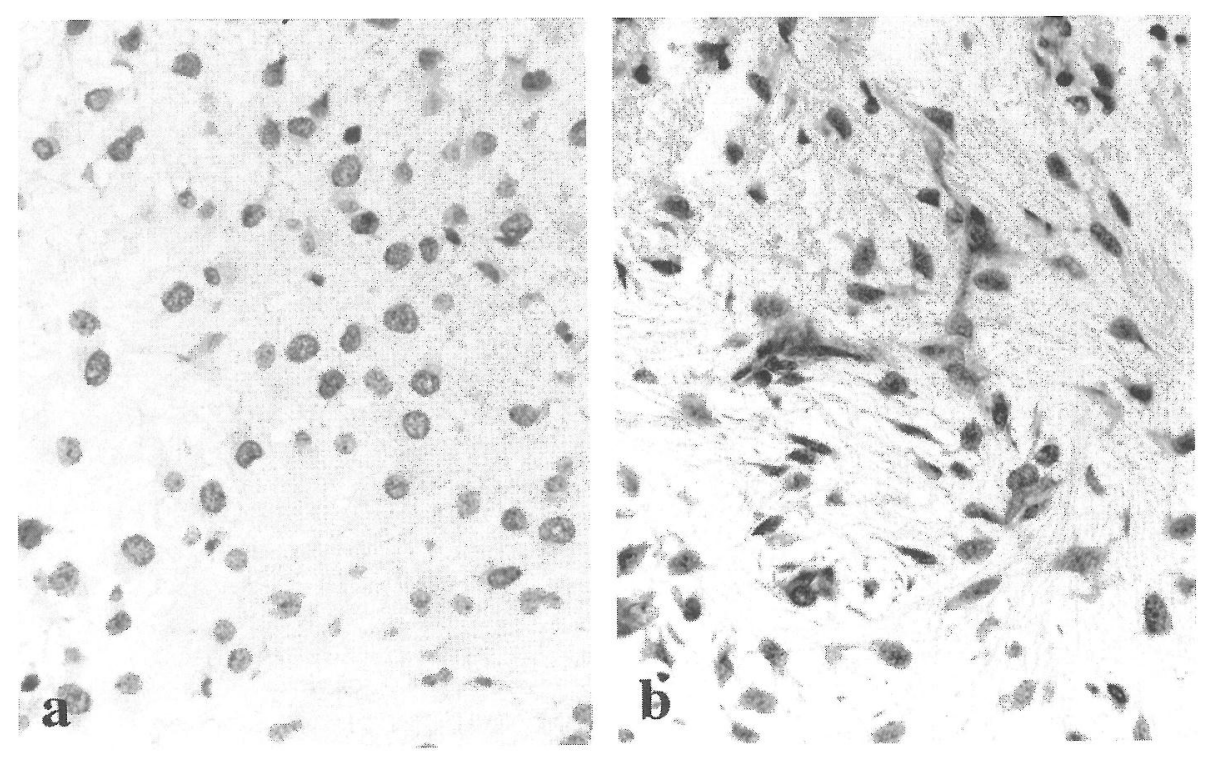

Fig. 3. TIMP-2 expression. (a) Negative staining of TIMP-2 both in immature teratoma and glial implants, and (b) positive in case without gliomatosis peritonei $($ TIMP-2, each $\times 200$ ). 
tissue and abundant immature glial tissues (Fig. 1a), accompanied by mature tissues composed of skin and adnexa, respiratory epithelium, and cartilage. All of the peritoneal implants were composed of mature glial tissues (grade 0) (Fig. 1b) surrounded by focal fibroblastic and chronic inflammatory reaction. The histology of the two cases of ovarian teratoma without gliomatosis peritonei was identical in that they contained immature glial component (grade 2, respectively) (Fig. 1c).

\section{Expression of MMPs and TIMPs}

The results of the immunostaining for the expressions of MMPs and TIMPs on glial cells which presented positive staining for GFAP are summarized in Table 1. The primary ovarian teratoma with glial implants (Fig. 2a) and the glial implants themselves (Fig. 2b) showed a high expression of MMP-1 and weak reaction of MMP-2 in the GFAP-positive glial tissues. In the two cases of immature teratoma without glial implants, expression of MMP-1 was seen (Fig. 2c) but MMP-2 was negative. In contrast, the expression of TIMP-2 was completely absent in the primary as well as glial implants (Fig. 3a) but was positive in the cases without gliomatosis peritonei (Fig. 3b). The expressions of MMP-3, MMP-9 and TIMP-1 were universally negative.

\section{Discussion}

An immature teratoma of the ovary gives rise to intraabdominal metastasis in up to one-third of the cases, most frequently as peritoneal or omental spread, with distinct prognostic and therapeutic implications [15]. In contrast, peritoneal or omental implants composed exclusively of mature (grade 0 ) glial tissue, aptly termed gliomatosis peritonei, is a rare event $[1,3-6,8,9,13,19]$. The capsular rupture of the tumor is thought to cause glial implants $[14,17,19]$. Even when the tumor capsule was not ruptured, pelvic adhesions or the adjacent localization of omentum to tumor capsule suggests a previous rupture [13]. In the present patient with gliomatosis peritonei, although the primary tumor was of $\mathrm{NBCH}$ size, a distinct rupture of the tumor or adhesive findings of omentum was not evident.

As summarized in Table 1, high MMP-1 expression was seen in the primary immature teratomas regardless of glial implants. Glial tissue accompanied the gliomatosis peritonei, and the gliomatosis peritonei itself presented a positive reaction to MMP-2, whereas the glial cells in the immature teratomas without gliomatosis were MMP-2 negative. The negative expression of TIMP-2 in the gliomatosis peritonei is in sharp contrast to the strong TIMP-2 expression in the immature teratomas without glial implants. Based on these results, gliomatosis peritonei may occur as the result of the ECM destruction caused by MMP-1 and partially by MMP-2 expression which may not be blocked by TIMP-2. In contrast, glial cell implants may be blocked by TIMP-2 expression in cases without gliomatosis peritonei. Although the biological significance of MMP-1, MMP-2 and TIMP-2 in gliomatosis peritonei is obscure $[12,16,20]$, it is highly possible that these enzymes (especially the loss of TIMP-2) play a crucial role in the glial dissemination of the development of gliomatosis peritonei. The further accumulation of cases is necessary to reach any definite conclusions.

\section{Acknowledgments}

This work was supported in part by a grant from the Japanese Association for Gynecologic Oncology and Chemotherapy. Presented at the Tenth International Congress of Histochemistry and Cytochemistory at Kyoto (1996).

\section{References}

1. Albites, V.: Solid teratoma of the ovary with malignant gliomatosis peritonei. Am. J. Obstet. Gynecol. 12; 59-65, 1974.

2. Apodoca, G., Rutka, J. T., Bouhana, K., Berens, M.E., Giblin, J. R., Rosenblum, M. L., Mckerrow, J. H. and Banda, M. J.: Expression of metalloproteinases and metalloproteinase inhibitors by fetal astrocytes and glioma cells. Cancer Res. 50; 2322-2329, 1990.

3. Bahari, C. M., Lurie, M., Schoenfeld, A. and Joel-Cohen, S. J.: Ovarian teratoma with peritoneal gliomatosis and elevated serum alpha-fetoprotein. Am. J. Clin. Pathol. 73; 603-607, 1980.

4. deGraaff, J. and van der Harten, J. J.: Alpha-fetoprotein in ovarian teratoma with glial implants on the peritoneum. Eur. J. Obstet. Gynaecol. Reprod. Biol. 10; 335-341, 1980.

5. Favara, B. E. and Franciosi, R. A.: Ovarian teratoma and neurogical implants on the peritoneum. Cancer 31; 678-681, 1973.

6. Fortt, R. W. and Mathie, I. K.: Gliomatosis peritonei caused by ovarian teratoma. J. Clin. Pathol. 22; 348-353, 1969.

7. Halaka, A. N., Bunning, R. A. D., Bird, C. C., Gibson, M. and Reynolds, J. J.: Production of collagenase and inhibitor (TIMP) by intracranial tumors and dura in vitro. $J$. Neurosurg. 59; 461-466, 1983.

8. Heydenrych, J. J., Villet, W. T. and du Toit, D. F.: Gliomatosis peritonei: The value of a "second look" operation. J. Surg. Oncol. 12; 119-125, 1979.

9. Khoo, S. K., Jones, I. S. C. and McKenna, H.: Ovarian teratoma with peritonel gliomatosis. Aust. NZ. J. Obstet. Gynaecol. 18; 277-280, 1978.

10. Lee, S., Iryo, O., Sugita, Y., Moriyama, T., Tokunaga, T. and Maruiwa, H.: Characterization of an established human malignant glioma cell line: KALS-1. Human Cell 3; 259-260, 1990.

11. Liotta, L. A., Rao, C. N. and Wewer, U. M.: Biochemical interactions of tumor cells with the basement membrane. Annu. Rev. Biochem. 55; 1037-1057, 1986.

12. Liotta, L. A., Steeg, P. S. and Stetler-Stevenson, W. G.: Cancer metastasis and angiogenesis: an imbalance of positive and negative regulation. Cell 64; 327-336, 1991.

13. Nielsen, S. N. J., Scheithauer, B. W. and Gaffey, T. A.: Gliomatosis peritonei. Cancer 56; 2499-2503, 1985.

14. Nogales, F. F. Jr. and Oliva, H. A.: Peritoneal gliomatosis produced by ovarian teratomas. Obstet. Gynecol. 43; 915920, 1974.

15. Norris, H. J., Zirken,' H. J. and Benson, W. L.: Immature 
(malignant) teratoma of the ovary. Cancer 37; 2359-2372, 1976.

16. Ogura, E., Senzaki, H., Kiyozuka, Y., Takada, H., Hioki, K. and Tsubura, A.: Expression and localization of matrix metalloproteinases and tissue inhibitors of metalloproteinases as a prognostic factor in advanced colorectal carcinomas. Oncol. Rep. 4; 701-705, 1997.

17. Robby, S. J. and Scully, R. E.: Ovarian teratoma with glial implants on the peritoneum: An analysis of 12 cases. Hum. Pathol. 1; 643-653, 1973.
18. Rutka, J. T., Giblin, J. R., Apodaca, G., DeArmond, S. J., Stein, R. and Rosenblum, M. L.: Inhibition of growth and induction of differentiation in a malignant human glioma cell line by normal leptomeningeal extracellular matrix proteins. Cancer Res. 47; 3515-3522, 1987.

19. Truong, L. D., Jurco, III. S. and McGavran, M. H.: Gliomatosis peritonei: Report of two cases and review of literature. Am. J. Surg. Pathol. 6; 443-449, 1982.

20. Woessner, J. F. Jr.: The family of matrix metalloproteinases. Ann. N.Y. Acad. Sci. 732; 11-21, 1994. 